\title{
The influence of the distribution of sound absorbing materials on the estimation of reverberation time in rooms
}

\author{
Marcelina Olechowska ${ }^{1, *}$, Artur Nowoświat ${ }^{1}$, Jan Ślusarek ${ }^{1}$ and Mateusz Latawiec ${ }^{2}$ \\ ${ }^{1}$ Silesian University of Technology, Faculty of Civil Engineering, Department of Building \\ Engineering and Building Physics, Akademicka 5 Str., 44-100 Gliwice, Poland \\ ${ }^{2}$ Author of diploma dissertation at the Silesian University of Technology, Faculty of Civil \\ Engineering, Department of Building Engineering and Building Physics, Akademicka 5 Str., \\ 44-100 Gliwice, Poland
}

\begin{abstract}
Reverberation time in rooms depends on many factors, e.g. cubature, surface of envelopes, sound absorption coefficient of materials used for the construction of the envelopes, geometry of rooms or the distribution of sound absorbing materials. The arrangement of sound absorbing materials in rooms has an impact on the dispersion of acoustic field, yet theoretical calculation models do not take into account this impact. According to these models, regardless of the arrangement of sound absorbing materials, the reverberation time in a room will remain unchanged. The present paper investigates the above problem by means of computer simulations. For the needs of the simulation, three rooms with different dimensions were adopted, i.e. type ' $\mathrm{p}$ ' - a cuboidal room with a square base, type ' $d$ ' - a cuboidal room (with one side of the ' $p$ ' room lengthened), type ' $w$ ' - a cuboidal room (with the height of the room lengthened ' $p$ '). During the simulation, the way of acoustic field dispersion was being changed and its influence on the reverberation time in the rooms was being determined. The authors investigated two situations. The first one involved a non-dampened room, in which the sound absorbing material was being arranged differently. The second one involved a welldampened room, and the dispersion of sound field was analyzed depending on the location of the reflecting material.
\end{abstract}

\section{Introduction}

Reverberation time plays a significant role in the design of school rooms [1], sacral rooms [2-4], auditoriums [5], theaters and concert halls [6], open-plan spaces [7], or others. This parameter has been investigated in many different works, a few of them mentioned below in the last years [8-10]. The measurement results of reverberation time can be applied to determine the sound absorption coefficient of sound absorbing materials in the reverberation chamber [11]. One of the factors which affects the reverberation time is the manner in which sound is dispersed in the room [12]. Irregular distribution of sound

*Corresponding author: marcelina.olechowska@polsl.pl 
absorbing materials in the room also affects the results obtained by means of various theoretical models. There are many available models [13], and each of them applies to rooms having various level of acoustic field dispersion. Therefore, a model for the estimation of reverberation time taking into account all known theoretical methods was developed [14]. As indicated in many works, inhomogeneous absorption [15-16], or room geometry deviating from the cubical shape can significantly affect the estimation of reverberation time. Ducourneau et al. [17] generalized the numerical method of acoustic field dispersion in a room. Also Billon et al. [18] investigated variable boundary conditions of the diffusion model. This study presents the characteristics of reverberation in three different rooms in which two different acoustic absorption variants were proposed. The investigated rooms were as follows: cuboidal room with a square base ' $p$ ', 'd' long, 'w' high. In each of the rooms two main variants were considered. In the first variant, the room was poorly dampened, and the location of sound absorbing material was being changed. The second variant involved a well dampened room in which the location of the reflecting material was being changed. Due to the nature of the work and a very large number of analyzed options, the tests were carried out in the computer programs ODEON and EASY.

\section{Method}

In the present work, the reverberation time RT was estimated for three types of rooms:

- Simulation of RT using the ODEON Version 12.0 computer program,

- Simulation of RT using the EASE 4.4 (Enhanced Acoustic Simulator for Engineers) computer program

- Calculation of RT by Sabine's formula,

- Calculation of RT by Eyring's formula,

- Calculation of RT by Kuttruff's formula,

- Calculation of RT by Arau Puchades.

\subsection{Computer program Odeon}

The computer simulations were carried out using the ODEON Room Acoustic program [19]. As written by Kostek in his work [20] „It is based on the hybrid method, which calculates the early reflections using a combination of the image source method and ray tracing, while the late reflections are calculated by a special ray tracing process generating diffuse secondary sources".

\subsection{Computer program Easy}

The application makes use of Sabine's and Eyring's formulas to calculate the reverberation time, and on that basis it calculates further parameters defined in the Standard ISO 3382 as well as speech intelligibility parameters (e.g.\% ALC, RASTI). The ray method is used to raise the accuracy of statistical methods and as a calculation method of the impulse response. The method consists in sending numbered rays from the sound source. Since the rays run through the whole room, the calculation algorithm tracks their duration time and energy carried at any moment. For each ray, we calculate the time after which the level of its energy is reduced by $60 \mathrm{~dB}$. Ultimately, these times are averaged for all traced rays and on that basis the reverberation time is estimated. 


\subsection{Theoretical models}

The existing theoretical models for the estimation of reverberation time are based on the statistical method, and by its application we eventually obtain the following formula:

$$
T_{S A B}=\frac{0.161 V}{S \bar{\alpha}}[s]
$$

with:

- For Sabine's model $\bar{\alpha}=\bar{\alpha}_{S A B}=\frac{1}{S} \sum_{i=1}^{n} \alpha_{i} S_{i}$

- For Eyring's model $\bar{\alpha}=\bar{\alpha}_{E u r}=-\ln \left(1-\bar{\alpha}_{S A B}\right)$

- For Arau Puchades' model

$$
\bar{\alpha}_{A R A U, x}=-\ln \left(1-\bar{\alpha}_{x}\right), \quad \bar{\alpha}_{A R A U, y}=-\ln \left(1-\bar{\alpha}_{y}\right), \quad \bar{\alpha}_{A R A U, z}=-\ln \left(1-\bar{\alpha}_{z}\right)
$$

And the reverberation time is calculated as:

$$
T_{A R A U}=\left[\frac{0.161 V}{S \bar{\alpha}_{A R A U, x}}\right]^{\frac{S_{x}}{S}} \cdot\left[\frac{0.161 V}{S \bar{\alpha}_{A R A U, y}}\right]^{\frac{S_{y}}{S}} \cdot\left[\frac{0.161 V}{S \bar{\alpha}_{A R A U, z}}\right]^{\frac{S_{z}}{S}}
$$

where: $S_{x}, S_{y}, S_{z}$ - surfaces of opposite walls $\left[\mathrm{m}^{2}\right]$,

$\bar{\alpha}_{x}, \bar{\alpha}_{y}, \bar{\alpha}_{z}$ - average reverberant sound absorption coefficients of the material on respective pairs of walls.

- For the Kuttruff's model $\bar{\alpha}=\bar{\alpha}_{K U T}=-\ln \left(1-\bar{\alpha}_{S A B}\right)\left(1+\frac{\gamma^{2}}{2} \ln \left(1-\bar{\alpha}_{S A B}\right)\right)$, where $\gamma^{2}$ stands for mean free path.

\subsection{Models of Rooms}

Three types of rooms were investigated, in which the measuring points were marked with blue color and different locations of the sound source in red. (Fig.1).

The cuboidal room was marked as ('p'), the room with the width and length as in ('p') but higher was marked as ('w') and the room with the width and height like in ('p') but longer was marked as ('d'). The dimensions of the rooms are presented in Table 1.

Table 1. Dimensions of the investigated rooms.

\begin{tabular}{|l|c|c|c|}
\hline Room & Width $[\mathbf{m}]$ & Length $[\mathbf{m}]$ & Height $[\mathbf{m}]$ \\
\hline Basic ('p') & 9 & 9 & 3 \\
\hline Longer ('d') & 9 & 27 & 3 \\
\hline Higher ('w') & 9 & 9 & 7 \\
\hline
\end{tabular}




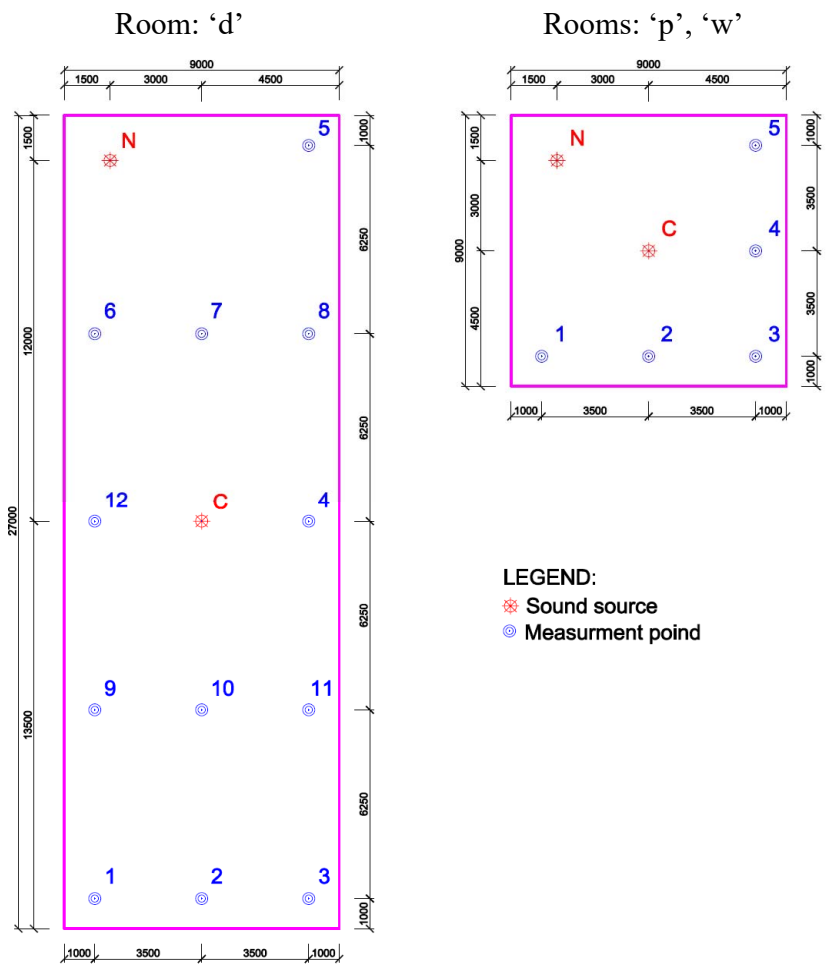

Fig. 1. Plans of the investigated rooms together with the reception points and sound sources.

Each of the rooms was investigated in two variants. Variant I - poorly dampened rooms, in which the sound absorbing material was placed in various ways and whereof area occupied $1 / 3$ of the shorter wall area. Variant II - well-dampened rooms, in which the reflective material was arranged in various ways and whereof area occupied $1 / 3$ of the shorter wall area, Thus, for the rooms of type ('p') and type ('d'), the sound absorbing and, respectively, sound reflecting material had the area of $9 \mathrm{~m}^{2}$, whereas for the rooms of type ('w') it was $21 \mathrm{~m}^{2}$. Table 2 presents sound absorption coefficients of the rooms in line with the variants I and II.

Table 2. Sound absorption coefficients of the applied materials.

\begin{tabular}{|c|c|c|c|c|c|c|c|c|}
\hline \multicolumn{10}{|c|}{ Variant I - poorly dampened room } \\
\hline \multicolumn{10}{|c|}{ basic material } \\
\hline $\mathrm{f}(\mathrm{Hz})$ & 63 & 125 & 250 & 500 & 1000 & 2000 & 4000 & 8000 \\
\hline$\alpha_{\mathrm{I}}$ & 0.02 & 0.02 & 0.02 & 0.03 & 0.04 & 0.05 & 0.05 & 0.05 \\
\hline \multicolumn{10}{|c|}{ added (distributed) material } \\
\hline $\mathrm{f}(\mathrm{Hz})$ & 63 & 125 & 250 & 500 & 1000 & 2000 & 4000 & 8000 \\
\hline$\alpha_{\mathrm{p}}$ & 0.45 & 0.45 & 0.85 & 0.99 & 0.99 & 0.99 & 0.99 & 0.99 \\
\hline \multicolumn{10}{|c|}{ ariant II - well-dampened room } \\
\hline \multicolumn{10}{|c|}{ basic material } \\
\hline $\mathrm{f}(\mathrm{Hz})$ & 63 & 125 & 250 & 500 & 1000 & 2000 & 4000 & 8000 \\
\hline$\alpha_{\mathrm{II}}$ & 0.12 & 0.12 & 0.09 & 0.33 & 0.33 & 0.44 & 0.57 & 0.62 \\
\hline \multicolumn{10}{|c|}{ added (distributed) material } \\
\hline $\mathrm{f}(\mathrm{Hz})$ & 63 & 125 & 250 & 500 & 1000 & 2000 & 4000 & 8000 \\
\hline$\alpha_{\mathrm{O}}$ & 0.02 & 0.02 & 0.02 & 0.03 & 0.04 & 0.05 & 0.05 & 0.05 \\
\hline
\end{tabular}


For the variant I, $\alpha_{I}$ stands for sound absorption coefficient of all envelopes limiting the room while $\alpha_{\mathrm{p}}$ stands for the absorbing material which was distributed accordingly. For variant II, $\alpha_{\text {II }}$ stands for sound absorption coefficient of all envelopes limiting the room, whereas $\alpha_{O}$ stands for the reflecting material which was distributed accordingly.

\section{Results and discussion}

Let us consider options in line with the variants I and II.

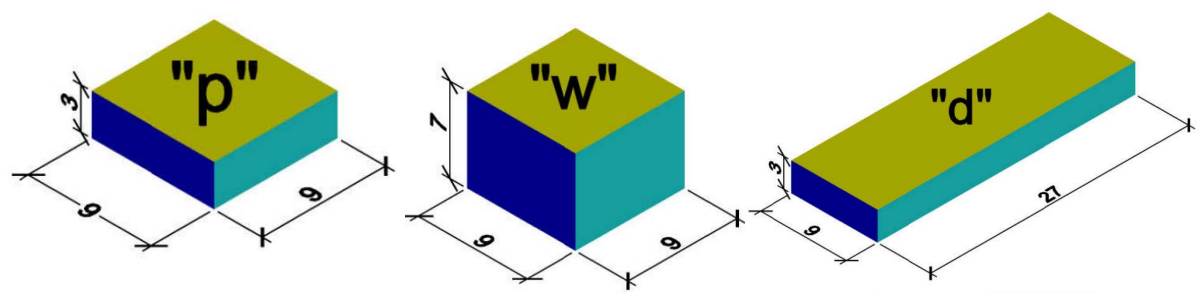

Fig. 2. Investigated rooms: "p" room with a square base 9x9m and height $3 \mathrm{~m}$, "w" changed height in relation to "p", "d" changed length in relation to "p".

The graphs in Figure 3 present the results of simulations and theoretical calculations for the room type "p" Fig. 2a) in variant I. The results involve the reverberation time for different arrangements of sound absorbing material.

a)

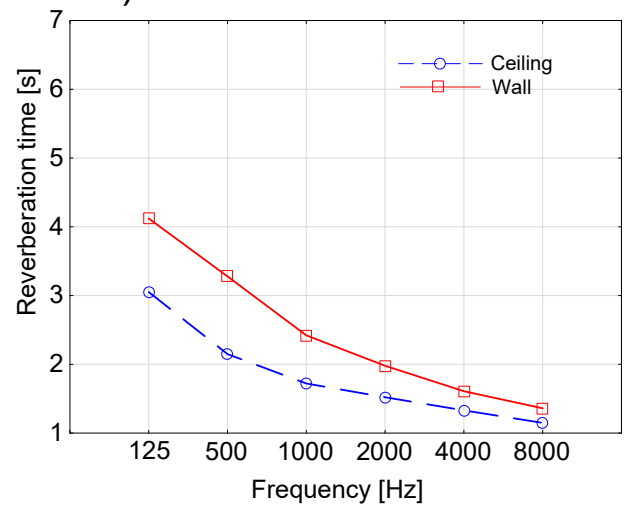

b)

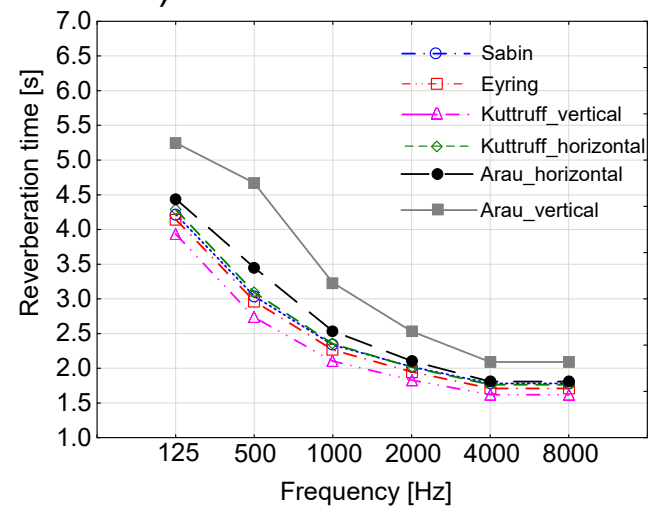

Fig. 3. a) results of ODEON simulation, b) theoretical results.

It turned out during the simulation that regardless of whether the sound absorbing material was placed on the ceiling or on the floor, the reverberation time did not change significantly. Similarly, it was irrelevant on which wall the sound absorbing material was located. However, the difference in reverberation time occurred depending on the location of the sound absorbing material on the horizontal or vertical envelope. In the theoretical calculations with the use of Sabine's and Eyring's methods, the reverberation time was always the same, regardless of the location of sound absorbing material. However, for Arau's and Kuttruff's models such differences were noticeable.

A similar analysis can be made for type "w" rooms Fig. 2 b. 
a)

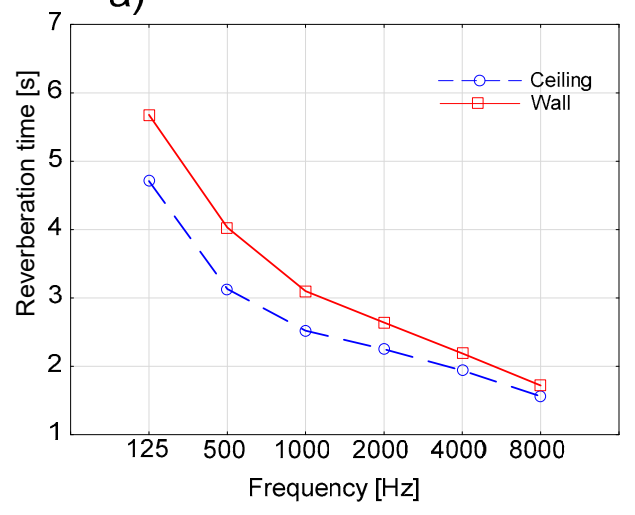

b)

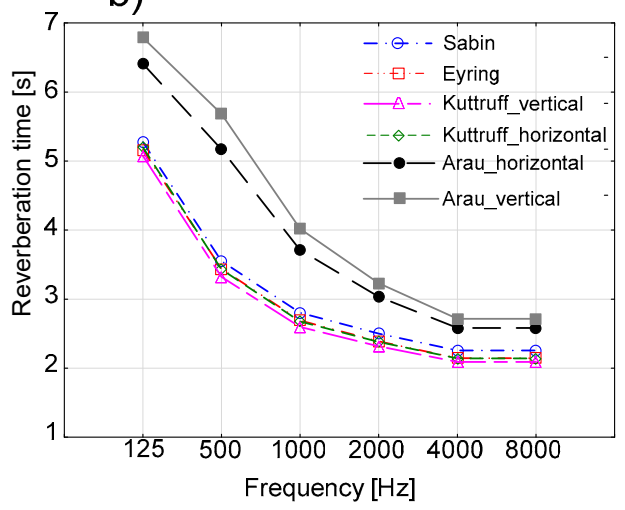

Fig. 4. a) results of ODEON simulation, b) theoretical results.

As before, it turned out during the simulation that regardless of whether the sound absorbing material was placed on the ceiling or on the floor, the reverberation time did not change significantly. Similarly, it was irrelevant on which wall the sound absorbing material was located. However, the difference in reverberation time occurred depending on the location of the sound absorbing material on the horizontal or vertical envelope. In the theoretical calculations with the use of Sabine's and Eyring's methods, the reverberation time was always the same, regardless of the location of sound absorbing material. However, for Arau's and Kuttruff's models, such differences were noticeable.

As in the previous cases, a similar analysis can be made for the type "d" room, Fig. 2c.

a)

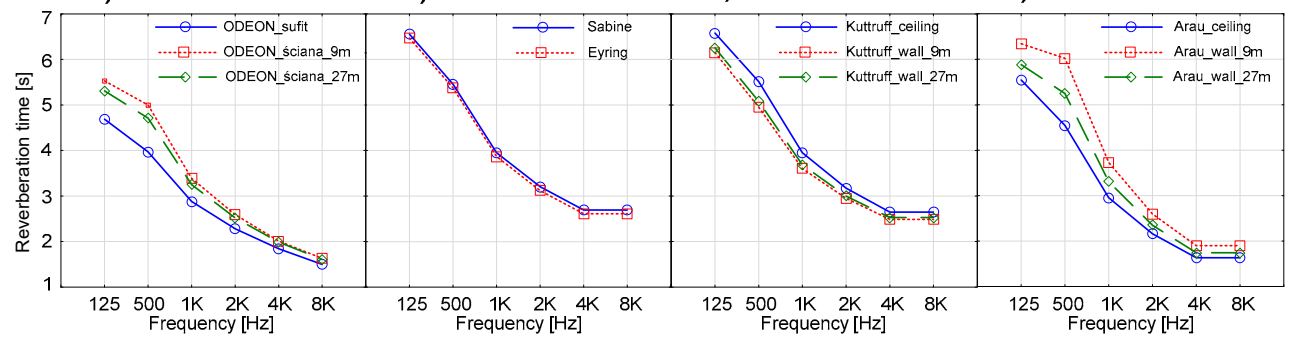

Fig. 5. a) results of ODEON simulation b) theoretical results of Sabine's, Eyring's, c) theoretical results of Kuttruff's, d) theoretical results of Arau-Puchades'.

For the " $\mathrm{d}$ " room, the reverberation time differed evidently depending on where the sound absorbing material was located, and the Sabine's and Eyring's formulas do not distinguish between the arrangement methods of sound absorbing material. The results obtained by means of ODEON simulations differ from the calculation results yielded by theoretical models.

In addition, the numerical and theoretical analyses demonstrate that for the Variant II, where the room is well dampened, the reverberation times obtained from the simulation do not change depending on the distribution of the material. The said effect is noticeable for all rooms, i.e. type "p", "w" and "d". The calculations obtained using the theoretical models reflect the theory, i.e. the Sabine's formula does not prove effective for dampened rooms. The other models in this case do not differ significantly. There is of course a possibility that using the algorithm applied in the EASY program we can obtain different results than with the algorithm from the ODEON program. In order to verify the significance of the results, 
the distribution of material on the shorter wall was simulated in both programs for both variants. The results are presented in Figure 6.

a)

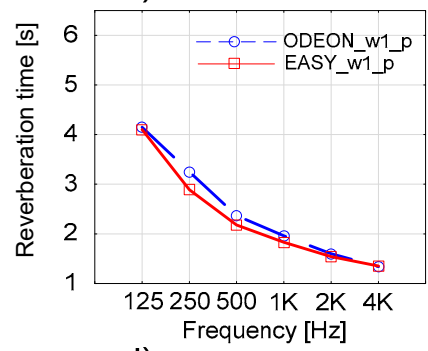

d)

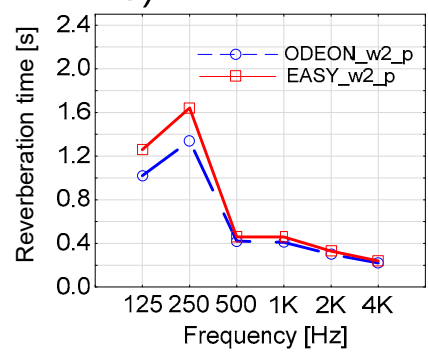

b)

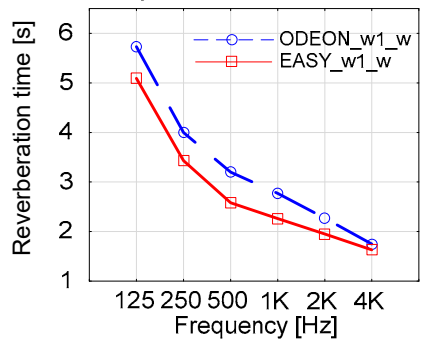

e)

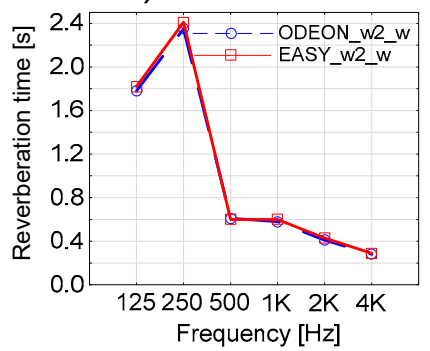

c)

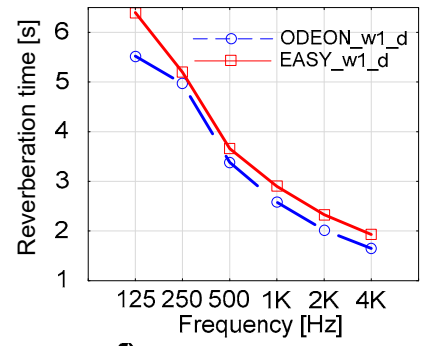

f)

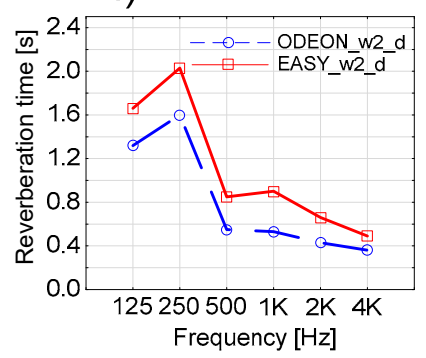

Fig. 6. Estimation results of the reverberation time using two different algorithms: ODEON, EASY. Graphs a, b, $\mathbf{c}$ show the simulations for variant I, and graphs $\mathbf{d}, \mathbf{e}, \mathbf{f}$ show the simulations for variant II.

As demonstrated in Figs. 6a, b, c, the reverberation times for poorly dampened rooms vary depending on the simulation algorithm. However, as demonstrated in Figs. 6d, e, f, for well dampened rooms, the reverberation times are estimated in a similar way, independently of the applied algorithm.

\section{Conclusion}

Based on the results of the simulation, the following conclusions can be drawn:

- The reverberation time in the room is affected not only by the absorbing surface area, but also by the arrangement of this surface. It is most apparent in poorly dampened rooms where sound absorbing surfaces are added.

- The Sabine's statistical model and its correction according to Eyring do not differentiate the reverberation time from the location of sound absorbing material.

- The Kuttruff's model, which takes into account the mean free path, differentiates the reverberation times depending on the location of sound absorbing material. Similarly, the differentiation occurs in the Arau-Puchades' model, which is a modification of the Fritzroy's model [13] and which is based on the calculations of reverberation time along three axes of the cuboidal room.

- The differences in the applied ODEON and EASY calculation algorithms have an impact on the values of reverberation time. However, they do not affect the nature of reverberation time changes as a function of frequency. The invariability of this character is maintained for all model rooms and different locations of the sound absorbing material. 


\section{References}

1. S.R., Bistafa, J.S. Bradley, J. Acoust. Soc. .Am. 108, 1721-1731 (2000)

2. U. Berardi, E. Cirillo, F. Martellotta, J. Acoust. Soc. .Am. 126, 1838 (2009)

3. U. Berardi, Joirnal of Building Performance Simulation, 7(1), 1-16 (2014)

4. K. Kosała, Arch. Acoust., 41(3), 458-498 (2016)

5. A. Gramez., F. Boumenider, Appl. Acoust., 118, 39-49 (2017)

6. T. Kamisiński, A. Kulowski, R. Kinasz, Arch. Acoust.,41(1), 3-14 (2016)

7. R. Golmohammadi, M. Aliabadi, T Nezami,Arch. Acoust., 42(2), 333-347 (2017)

8. H. Arau-Puchades, U. Berardi, Arch. Acoust. 40(1), 33-40 (2015)

9. M. Olechowska, J. Ślusarek, Architecture Civil Engineering Environmental ACEE, 4, 79-87 (2016)

10. V.G. Escobar, J.M. Barringón Morillas, Appl. Acoust. 96, 1-10 (2015)

11. A. Nowoświat, J. Bochen, L. Dulak, R. Żuchowski, Appl Acoust., 111, 8-15 (2016)

12. A. Prato, F. Casassa, A. Schiavi, Appl. Acoust., 110, 160-169 (2016)

13. A. Nowoświat, M. Olechowska, Arch. Acoust., 41(1), 15-26 (2016)

14. A. Nowoświat, M., Olechowska, J. Ślusarek, Appl. Acoust., 106, 42-50 (2016)

15. M. Hodgson, Appl. Acoust., 49(3), 197-207 (1996)

16. S.R. Bistafa, J.S. Bradley, J. Acoust. Soc. Am., 108(4), 1721-1731 (2000)

17. J. Ducoirneau, V. Planeau, Appl. Acoust. 64(9), 845-862 (2003)

18. A. Billon, J. Picaut, A. Sakout, Appl. Acoust. 69, 68-74 (2008)

19. I. Bork, Acta Acustica, 86, 943-956 (2000)

20. B. Kostek, S. Laskowski, K. Mizgier, Arch. Acoust., 41(3), 473-484 (2016) 